\title{
KOSZUL DUALITY AND GALOIS COHOMOLOGY
}

\author{
LeOnid Positselski And Alexander VishiK
}

\section{Introduction}

Let $F$ be a field, $\bar{F}$ be its (separable) algebraic closure, and $G_{F}=$ $\operatorname{Gal}(\bar{F} / F)$ be the absolute Galois group. Let $l \neq \operatorname{char} F$ be a prime number; assume that $F$ contains a $l$-root of unity $\zeta$. In this case, the Kummer pairing

$$
\kappa: G_{F} \times F^{*} \longrightarrow \mathbb{F}_{l}, \quad \kappa(g, a)=s \text { if } g(b)=\zeta^{s} b \text { for } b=\sqrt[l]{a} \in \bar{F}
$$

defines an isomorphism $F^{*} /\left(F^{*}\right)^{l} \simeq H^{1}\left(G_{F}, \mathbb{F}_{l}\right)$. The Milnor K-theory ring $K^{\mathrm{M}}(F)$ is a skew-commutative quadratic algebra over $\mathbb{Z}$ generated by $K_{1}^{\mathrm{M}}(F)=F^{*}$ with the Steinberg relations $\{a, 1-a\}=0$. It is not difficult to show that the Kummer map can be extended to an algebra homomorphism

$$
K^{\mathrm{M}}(F) \otimes \mathbb{F}_{l} \longrightarrow H^{*}\left(G_{F}, \mathbb{F}_{l}\right),
$$

which is known as the Galois symbol, or the norm residue homomorphism. The well-known Bloch-Kato conjecture claims that it is an isomorphism. It was proved by A. Merkurjev and A. Suslin [10, 11] and M. Rost [16] that this is true in degree 2 and for $l=2$ in degree 3 . The aim of this note is to show that the whole conjecture follows from its low-degree part provided the quadratic algebra $K^{\mathrm{M}}(F) \otimes \mathbb{F}_{l}$ is Koszul (see section 2 for the definition). We will assume that $F$ has no algebraic extensions of degree relatively prime to $l$.

Theorem. Let $H=H^{*}\left(G, \mathbb{F}_{l}\right)$ be the cohomology algebra of a pro-l-group G. Assume that

(1) $H^{2}$ is generated by $H^{1}$;

(2) in the subalgebra generated by $H^{1}$ in $H$, there are no nontrivial relations of degree 3 ;

(3) the quadratic algebra defined by $H^{1}$ and $H^{2}$ is Koszul.

Then the whole algebra $H$ is quadratic.

Received September 21, 1995. 
Actually, it is not essential that we consider the group cohomology here; the theorem is valid for any pro-nilpotent algebra. We will use the language of coalgebras in this paper in order to avoid dealing with projective limits and dualizations.

\section{Bar construction}

1.1. The cohomology of augmented coalgebras. A coalgebra is a vector space $C$ over a field $\mathbb{k}$ equipped with $\Delta: C \longrightarrow C \otimes C$, a comultiplication map, and $\varepsilon: C \longrightarrow \mathbb{k}$, a counit map satisfying the conventional associativity and counit axioms. An augmented coalgebra is a coalgebra $C$ equipped with a coalgebra homomorphism $\gamma: \mathbb{k} \longrightarrow C$. The cohomology algebra of an augmented coalgebra $C$ is defined as the Ext-algebra $H^{*}(C)=\operatorname{Ext}_{C}^{*}(\mathbb{k}, \mathbb{k})$ in the category of left $C$-comodules, where $\mathbb{k}$ is endowed with the comodule structure by means of $\gamma$. We will calculate this cohomology using the explicit comodule resolution

$$
\mathbb{k} \longrightarrow C \longrightarrow C \otimes C^{+} \longrightarrow C \otimes C^{+} \otimes C^{+} \longrightarrow \cdots,
$$

where $C^{+}=\operatorname{coker}(\gamma)$, the differential is

$$
d\left(c_{0} \otimes \cdots \otimes c_{n}\right)=\sum_{i=0}^{n}(-1)^{i-1} c_{0} \otimes \cdots \otimes \Delta\left(c_{i}\right) \otimes \cdots \otimes c_{n}
$$

and the coaction of $C$ is through the left components of these tensors. It is easy to check that the comodules $C \otimes W$ are injective, the differential is well-defined, and the operator

$$
h: c_{0} \otimes c_{1} \otimes \cdots \otimes c_{n} \longmapsto \varepsilon\left(c_{0}\right) \sigma\left(c_{1}\right) \otimes c_{2} \otimes \cdots \otimes c_{n},
$$

where $\sigma: C^{+} \longrightarrow C$ is the splitting along $\varepsilon$, provides a $\mathbb{k}$-linear contracting homotopy. Thus this is a resolution. Applying the functor $\operatorname{Hom}_{C}(\mathbb{k}, \cdot)$, we obtain

$$
H^{*}(C)=H^{*}\left(\mathbb{k} \longrightarrow C^{+} \longrightarrow C^{+} \otimes C^{+} \longrightarrow \cdots\right),
$$

where the differential is given by the same formula and the multiplication on $H^{*}(C)$ is induced by the evident multiplication

$$
\left(c_{1} \otimes \cdots \otimes c_{i}\right) \cdot\left(c_{i+1} \otimes \cdots \otimes c_{i+j}\right)=c_{1} \otimes \cdots \otimes c_{i} \otimes c_{i+1} \otimes \cdots \otimes c_{i+j}
$$

on this cobar-complex. 
1.2. The homology of augmented algebras. An augmented algebra $A$ is an associative algebra over a field $\mathbb{k}$ endowed with an algebra homomorphism $\alpha: A \longrightarrow \mathbb{k}$. The homology coalgebra of an augmented algebra $A$ is by the definition $H_{*}(A)=\operatorname{Tor}_{*}^{A}(\mathbb{k}, \mathbb{k})$, where the left and right module structures on $\mathbb{k}$ are defined by means of $\alpha$. We will calculate it using the following explicit bar-resolution of the left $A$-module $\mathbb{k}$

$$
\mathbb{k} \longleftarrow A \longleftarrow A \otimes A_{+} \longleftarrow A \otimes A_{+} \otimes A_{+} \longleftarrow \cdots,
$$

where $A_{+}=\operatorname{ker}(\alpha)$ and

$$
\partial\left(a_{0} \otimes \cdots \otimes a_{n}\right)=\sum_{i=1}^{n}(-1)^{i} a_{0} \otimes \cdots \otimes a_{i-1} a_{i} \otimes \cdots \otimes a_{n} .
$$

It is easy to check that the operator

$$
h: a_{0} \otimes a_{1} \otimes \cdots \otimes a_{n-1} \longmapsto 1 \otimes\left(a_{0}-\alpha\left(a_{0}\right)\right) \otimes a_{1} \otimes \cdots \otimes a_{n-1}
$$

provides a $\mathbb{k}$-linear contracting homotopy. Applying the functor $\mathbb{k} \otimes_{A} \cdot$, we obtain

$$
H_{*}(A)=H_{*}\left(\mathbb{k} \longleftarrow A_{+} \longleftarrow A_{+} \otimes A_{+} \longleftarrow \cdots\right)
$$

and the coalgebra structure on $H_{*}(A)$ is induced by the evident coalgebra structure

$$
\Delta\left(a_{1} \otimes \cdots \otimes a_{n}\right)=\sum_{i=0}^{n}\left(a_{1} \otimes \cdots \otimes a_{i}\right) \otimes\left(a_{i+1} \otimes \cdots \otimes a_{n}\right)
$$

on this bar-complex.

\section{Koszul duality}

A graded algebra (graded coalgebra) is a non-negatively graded vector space $A=\bigoplus_{n=0}^{\infty} A_{n}\left(C=\bigoplus_{n=0}^{\infty} C_{n}\right)$ over a field $\mathbb{k}$ such that $A_{0}=\mathbb{k}\left(C_{0}=\right.$ $\mathbb{k}$ ) which is equipped with an associative algebra (coalgebra) structure that respects the grading, i.e., $A_{i} \cdot A_{j} \subset A_{i+j}$ and $1 \in A_{0}\left(\Delta\left(C_{n}\right) \subset\right.$ $\sum_{i+j=n} C_{i} \otimes C_{j}$ and $\left.\varepsilon\left(C_{>0}\right)=0\right)$. A graded algebra (coalgebra) structure induces an augmented algebra (coalgebra) structure in an evident way. The homology coalgebra (cohomology algebra) of a graded algebra (coalgebra) is equipped with a natural second grading, as it can be seen from the explicit resolutions above:

$$
H_{*}(A)=\bigoplus_{i \leqslant j} H_{i j}(A) \quad \text { and } \quad H^{*}(C)=\bigoplus_{i \leqslant j} H^{i j}(C) .
$$

In fact, all the results below in this section can be formulated in a more general setting of a graded algebra in a (semisimple abelian, not necessarily 
symmetric) tensor category, where the duality connects the algebras in the opposite categories; however, we prefer to deal with vector spaces here.

\subsection{Quadratic algebras and coalgebras.}

Definition 1. A graded coalgebra $C$ is called one-cogenerated if the iterated comultiplication maps $\Delta^{(n)}: C_{n} \longrightarrow C_{1}^{\otimes n}$ are injective, or equivalently, all the maps $\Delta: C_{i+j} \longrightarrow C_{i} \otimes C_{j}$ are injective. A graded coalgebra is called quadratic if it is isomorphic to the subcoalgebra of the form

$$
\langle V, R\rangle=\bigoplus_{n=0}^{\infty} \bigcap_{i=1}^{n-1} V^{i-1} \otimes R \otimes V^{n-i-1}
$$

of the tensor coalgebra $\mathbb{N}(V)=\bigoplus_{n} V^{\otimes n}$ for some vector space $V$ and a subspace $R \subset V^{\otimes 2}$. With a graded coalgebra $C$, one can associate in a natural way a quadratic coalgebra $\mathrm{q} C$ and a morphism of graded coalgebras $r_{C}: C \longrightarrow \mathrm{q} C$ that is an isomorphism on $C_{1}$ and an epimorphism on $C_{2}$. A graded algebra is called quadratic if it is isomorphic to the quotient algebra $\{V, R\}=\mathbb{T}(V) /(R)$ of a tensor algebra $\mathbb{T}(V)=\bigoplus_{n} V^{\otimes n}$ by the ideal generated by a subspace $R \subset V^{\otimes 2}$. With a graded algebra $A$, one can associate a quadratic algebra $\mathrm{q} A$ and a morphism of graded algebras $r_{A}: \mathrm{q} A \longrightarrow A$ that is an isomorphism on $A_{1}$ and a monomorphism on $A_{2}$.

Definition 2. The quadratic algebra $A=\{V, R\}$ and the quadratic coalgebra $C=\langle V, R\rangle$ are called dual to each other; we denote this as $C=A^{\text {! }}$ and $A=C$ ? Evidently, this defines an equivalence between the categories of quadratic algebras and quadratic coalgebras.

Proposition 1. A graded coalgebra $C$ is one-cogenerated if and only if $H^{1, j}(C)=0$ for $j>1$. A one-cogenerated coalgebra $C$ is quadratic iff $H^{2, j}(C)=0$ for $j>2$. Moreover, the morphism $r_{C}: C \longrightarrow \mathrm{q} C$ is an isomorphism on $C_{\leqslant n}$ iff $H^{2, j}(C)=0$ for $2<j \leqslant n$. The analogous statements are true for graded algebras.

Proof. The explicit form of the cobar-complex implies that $H^{1,>1}(C)=0$ for a one-cogenerated coalgebra $C$. Conversely, let $j>1$ be the minimal number for which $\Delta^{(j)}: C_{j} \longrightarrow C_{1}^{\otimes j}$ is non-injective, then it is easy to see that the map $\Delta: C_{j} \longrightarrow \bigoplus_{s, t \geqslant 1}^{s+t=j} C_{s} \otimes C_{t}$ is non-injective also, hence $H^{1, j}(C) \neq 0$. Now let $C$ be one-cogenerated, then the map $r_{C}$ is an embedding. Let $z \in C_{+} \otimes C_{+}$be a homogeneous cocycle of degree $n$, thus $z=\sum_{s, t \geqslant 1}^{s+t=n} z_{s t}$, where $z_{s t} \in C_{s} \otimes C_{t}$. The cocycle condition means that the images of $(\Delta \otimes \mathrm{id})\left(z_{u+v, w}\right)$ and $(\mathrm{id} \otimes \Delta)\left(z_{u, v+w}\right)$ in $C_{u} \otimes C_{v} \otimes C_{w}$ coincide for any $u, v, w \geqslant 1, u+v+w=n$. Since the maps $\Delta^{(k)}$ are injective, it is equivalent to say that the elements $\left(\Delta^{(s)} \otimes \Delta^{(t)}\right)\left(z_{s t}\right) \in C_{1}^{\otimes n}$ coincide for all $s$ and $t$. We have got an element in $C_{1}^{\otimes n}$; it is easy to see 
that it represents an element of qC which belongs to the image of $r_{C}$ iff $z$ is a coboundary. At last, if $r_{C}$ is an isomorphism in degree $<n$, then any element of $\mathrm{q} C$ corresponds to a cocycle $z$ in this way.

Proposition 2. For any graded coalgebra $C$, the diagonal subalgebra $\bigoplus_{i} H^{i, i}(C)$, of the cohomology algebra $H^{*}(C)$, is a quadratic algebra isomorphic to $(\mathrm{q} C)^{\text {? }}$. Analogously, for a graded algebra $A$, the diagonal quotient coalgebra $\bigoplus_{i} H_{i, i}(A)$ of the homology coalgebra $H_{*}(A)$ is isomorphic to $(\mathrm{q} A)^{!}$.

Proof. Immediate.

2.2. Koszul algebras and coalgebras. The following definition is due to S. Priddy [15] (see also [8, 1, 2, 4]):

Definition 3. A graded algebra $A$ is called Koszul if $H_{i j}(A)=0$ unless $i=j$. A graded coalgebra $C$ is called Koszul if $H^{i j}(C)=0$ unless $i=$ $j$. It follows from Proposition 1 that any Koszul algebra (coalgebra) is quadratic.

Now we are going to establish the criterion of Koszulity in the explicit linear algebra terms due to J. Backelin [1]. In particular, we will see that the dual algebra and coalgebra are Koszul simultaneously.

Definition 4. A collection of subspaces $X_{1}, \ldots, X_{n-1}$ in a vector space $W$ is called distributive, if there exists a (finite) direct decomposition $W=$ $\bigoplus_{\omega \in \Omega} W_{\omega}$ such that each subspace $X_{k}$ is the sum of a set of subspaces $W_{\omega}$. Equivalently, the distributivity identity $(X+Y) \cap Z=X \cap Z+Y \cap Z$ should be satisfied for any triple of subspaces $X, Y, Z$ that can be obtained from the subspaces $X_{k}$ using the operations of sum and intersection.

Lemma. Let $X_{1}, \ldots, X_{n-1} \subset W$ be a collection of linear subspaces; assume that any proper subcollection $X_{1}, \ldots, \widehat{X}_{k}, \ldots, X_{n-1}$ is distributive. Then the following three conditions are equivalent:

(a) the following complex $B^{*}(W, X)$ is exact everywhere outside its left term:

$W \longrightarrow \bigoplus_{s} W / X_{s} \longrightarrow \bigoplus_{s<t} W /\left(X_{s}+X_{t}\right) \longrightarrow \cdots \longrightarrow W / \sum_{k} X_{k} \longrightarrow 0$;

(b) the following complex $B_{*}(W, X)$ is exact everywhere outside its left term:

$W \longleftarrow \bigoplus_{s} X_{s} \longleftarrow \bigoplus_{s<t} X_{s} \cap X_{t} \longleftarrow \cdots \longleftarrow \bigcap_{k} X_{k} \longleftarrow 0$

(c) the collection $X_{1}, \ldots, X_{n-1}$ is distributive.

Proposition 3. Let $V$ be a vector space and $R \subset V \otimes V$ be a subspace, then the following three conditions are equivalent: 
(a) the quadratic algebra $A=\{V, R\}$ is Koszul;

(b) the quadratic coalgebra $C=\langle V, R\rangle$ is Koszul;

(c) for any $n$, the collection of subspaces $V^{\otimes k-1} \otimes R \otimes V^{n-k-1} \subset V^{\otimes n}$, where $k=1, \ldots, n-1$, is distributive.

Proof. Moreover, one has $H_{i j}(A)=0$ for $i<j \leqslant n$ iff the collection of subspaces in $V^{\otimes n}$ is distributive, and the same for coalgebras. This follows immediately from Lemma by induction on $n$.

\section{Cohomology of nilpotent coalgebras}

3.1. Nilpotent coalgebras. Let $C$ be an augmented coalgebra with the augmentation map $\gamma: \mathbb{k} \longrightarrow C$. The augmentation filtration on an augmented coalgebra $C$ is an increasing filtration $N$ defined by the formula

$$
\begin{aligned}
& N_{n} C=\{c \in C \mid \\
& \left.\quad \Delta^{(n+1)}(c) \in C_{\gamma}^{\otimes n+1}=\sum_{i=1}^{n+1} C^{\otimes i-1} \otimes \gamma(\mathbb{k}) \otimes C^{\otimes n-i+1} \subset C^{\otimes n+1}\right\},
\end{aligned}
$$

where $\Delta^{(m)}: C \longrightarrow C^{\otimes m}$ denotes the iterated comultiplication map. In particular, we have $N_{0} C=\gamma(\mathbb{k})$.

Proposition 4. The filtration $N$ respects the coalgebra structure on $C$, that is

$$
\Delta\left(N_{n} C\right) \subset \sum_{i+j=n} N_{i} C \otimes N_{j} C .
$$

Furthermore, the associated graded coalgebra $\operatorname{gr}_{N} C=\bigoplus_{n=0}^{\infty} N_{n} C / N_{n-1} C$ is one-cogenerated.

Proof. Let $\phi: C \longrightarrow \mathbb{k}$ be a linear function annihilating $N_{k-1} C$, where $0 \leqslant k \leqslant n$; then it can be factorized as $\phi=\psi \circ \Delta^{(k)}$, where $\psi: C^{\otimes k} \longrightarrow \mathbb{k}$ is a function annihilating $C_{\gamma}^{\otimes k}$. We have to show that $(\phi \otimes \mathrm{id}) \Delta N_{n} C \subset$ $N_{n-k} C$. Put for convenience $\Delta^{(0)}=\varepsilon$ and $\Delta^{(1)}=\mathrm{id}$; then one has $\left(\Delta^{(k)} \otimes \Delta^{(n-k+1)}\right) \circ \Delta=\Delta^{(n+1)}$, hence

$$
\left(\phi \otimes \Delta^{(n-k+1)}\right) \Delta N_{n} C=\left(\psi \otimes \mathrm{id}^{\otimes n-k+1}\right) \Delta^{(n+1)} N_{n} C \subset C_{\gamma}^{\otimes n-k+1},
$$

so we are done. Since we have $\Delta^{(n)}(c) \notin C_{\gamma}^{\otimes n}$ for $c \notin N_{n-1} C$, the second assertion is immediate.

Definition 5. An augmented coalgebra $C$ is called nilpotent if the augmentation filtration $N$ is full, that is, if $C=\bigcup_{n} N_{n} C$. 
Example. Let $G$ be a pro-l-group and $C=\mathbb{F}_{l}(G)$ be the coalgebra of locally constant functions on $G$ with respect to the convolution; in other words, $C=\varliminf_{G} \mathbb{F}_{l}(G / U)$, where the limit is taken over all open normal subgroups $U$ of $\vec{G}$ and the coalgebra $\mathbb{F}_{l}(G / U)=\mathbb{F}_{l}[G / U]^{*}$ is the vector space dual to the group algebra of $G / U$. Let $\gamma: \mathbb{F}_{l} \longrightarrow \mathbb{F}_{l}(G)$ be the augmentation map that takes a constant from $\mathbb{F}_{l}$ to the corresponding constant function on $G$. Since the augmentation ideal of the group ring of a finite $l$-group over $\mathbb{F}_{l}$ is nilpotent, it follows by passing to the inductive limit that the augmented coalgebra $\mathbb{F}_{l}(G)$ is nilpotent also. It is easy to see that the category of $\mathbb{F}_{l}(G)$-comodules is equivalent to the category of discrete $G$-modules over $\mathbb{F}_{l}$ (and the same is true over $\mathbb{Z}$ ) for any pro-finite group $G$.

3.2. Main theorem. Now we are ready to prove the theorem mentioned in the introduction.

Theorem. Let $H=H^{*}(C)$ be the cohomology algebra of a nilpotent coalgebra $C$. Assume that

(1) $H^{2}$ is generated by $H^{1}$;

(2) in the subalgebra generated by $H^{1}$ in $H$, there are no nontrivial relations of degree 3 ;

(3) the quadratic algebra $\mathrm{q} H$ defined by $H^{1}$ and $H^{2}$ is Koszul.

Then the whole algebra $H$ is quadratic (and therefore, Koszul). In addition, there is an isomorphism $H^{*}(C) \simeq H^{*}\left(\operatorname{gr}_{N} C\right)$.

Proof. The filtration $N$ on a coalgebra $C$ induces a filtration on the corresponding cobar-complex:

$$
N_{n} C^{+\otimes i}=\sum_{j_{1}+\cdots+j_{i}=n} N_{j_{1}} C^{+} \otimes \cdots \otimes N_{j_{i}} C^{+}
$$

where $N_{j} C^{+}=N_{j} C / \gamma(\mathbb{k})$, so that the filtration on $C^{+\otimes i}$ starts with $N_{i}$. Clearly, the associated graded complex coincides with the cobar-complex of $\operatorname{gr}_{N} C$, thus we obtain a multiplicative spectral sequence

$$
E_{1}^{i j}=H^{i j}\left(\operatorname{gr}_{N} C\right) \Longrightarrow H^{i}(C)
$$

which converges since the filtration is an increasing one. More exactly, the differentials have the form $d_{r}: E_{r}^{i, j} \longrightarrow E_{r}^{i+1, j-r}$ and there is an induced increasing multiplicative filtration $N$ on $H^{*}(C)$ such that $\operatorname{gr}_{N}^{j} H^{i}(C)=$ $E_{\infty}^{i, j}$.

In particular, we see that the subalgebra $\bigoplus N_{i} H^{i}(C)$ in $H^{*}(C)$ is isomorphic to the quotient algebra of the diagonal cohomology $\bigoplus H^{i, i}\left(\operatorname{gr}_{N} C\right)$ 
by the images of the differentials. By Proposition 4, the graded coalgebra $\operatorname{gr}_{N} C$ is one-cogenerated, hence (by Proposition 1) we have $E_{1}^{1, j}=$ $H^{1, j}\left(\operatorname{gr}_{N} C\right)=0$ for $j>1$, which implies that

$$
H^{1}(C)=N_{1} H^{1}(C) \simeq H^{1,1}\left(\operatorname{gr}_{N} C\right) \quad \text { and } \quad N_{2} H^{2}(C) \simeq H^{2,2}\left(\operatorname{gr}_{N} C\right) .
$$

Since (by Proposition 2) the diagonal cohomology algebra $\bigoplus H^{i, i}\left(\operatorname{gr}_{N} C\right)$ is quadratic, we conclude that it is isomorphic to $\mathrm{q} H^{*}(C)$. By Proposition 2 again, $\bigoplus H^{i, i}\left(\operatorname{gr}_{N} C\right)$ is the dual quadratic algebra to the coalgebra $\operatorname{qgr}_{N} C$; since we suppose $\mathrm{q} H^{*}(C)$ is Koszul, the dual coalgebra $\operatorname{qgr}_{N} C$ is Koszul also (Proposition 3). On the other hand, we have assumed that there are no cubic relations in the subalgebra generated by $H^{1}(C)$, hence all the differentials $d_{r}: E_{r}^{2,3+r} \longrightarrow E_{r}^{3,3}$ targeting in $H^{3,3}\left(\operatorname{gr}_{N} C\right)$ vanish.

Now let us prove by induction that $H^{2, j}\left(\operatorname{gr}_{N} C\right)=0$ for $j>2$. Assume that this is true for $2<j \leqslant n-1$; by Proposition 1, it follows that the map $r_{\operatorname{gr}_{N} C}: \operatorname{gr}_{N} C \longrightarrow \operatorname{qgr}_{N} C$ is an isomorphism in degree $\leqslant n-1$. Therefore, the induced map on the cobar-complex is an isomorphism in these degrees also, hence in particular $H^{3, j}\left(\operatorname{gr}_{N} C\right)=H^{3, j}\left(\operatorname{qgr}_{N} C\right)$ for $j \leqslant n-1$ (and even for $j \leqslant n$ ). Since the coalgebra $\operatorname{qgr}_{N} C$ is Koszul, it follows that $E_{1}^{3, j}=H^{3, j}\left(\operatorname{gr}_{N} C\right)=0$ for $3<j \leqslant n-1$ and the term $E_{1}^{2, n}=H^{2, n}\left(\operatorname{gr}_{N} C\right)$ cannot die in the spectral sequence. But we have assumed that $H^{2}(C)$ is generated by $H^{1}(C)$. Hence $H^{2}(C)=N_{2} H^{2}(C)$ and $E_{\infty}^{2, n}=0$; so we are done.

We have seen that the coalgebra $\operatorname{gr}_{N} C$ is quadratic and $\operatorname{qgr}_{N} C$ is Koszul, that is, $\operatorname{gr}_{N} C$ is Koszul. It follows that $E_{1}^{i, j}=0$ for $i \neq j$, thus the spectral sequence degenerates and $H^{*}(C)=H^{*}\left(\operatorname{gr}_{N} C\right)$. Therefore, $H^{*}(C)$ is Koszul also.

Remark. This result is a formal analogue of a kind of Poincare-BirkhoffWitt theorem for filtrations on quadratic algebras [14]; in other words, it can be considered as reflecting the deformation properties of Koszul algebras.

In conclusion, recall the consequences we get for the Bloch-Kato conjecture. Since the conditions (1) and (2) of our Theorem are known to be satisfied for the coalgebra $C=\mathbb{F}_{2}\left(G_{F}\right)$ of any absolute Galois pro-2-group $G_{F}$ and the quadratic part $\mathrm{q} H^{*}(C)$ of the corresponding cohomology algebra is exactly the Milnor K-theory algebra $K^{\mathrm{M}}(F) \otimes \mathbb{F}_{2}$, it suffices to establish the Koszul property of this quadratic algebra in order to prove the conjecture for $l=2$. The same would be true for the other $l$ if we knew that the norm residue homomorphism for that $l$ is injective in degree 3 . 


\section{Appendix: $K^{\mathrm{M}}(F) \otimes \mathbb{F}_{l}$ is Koszul for all primitive fields $F$}

A.1. Commutative PBW-bases. Let $A$ be a commutative or skewcommutative one-generated algebra over a field $\mathbb{k}$. Fix a basis $\mathcal{X}=\{x\}$ of the vector space $A_{1}$; suppose $\mathcal{X}$ is equipped with a complete order $x^{\prime}<x^{\prime \prime}$, i.e., there are no infinite decreasing sequences $x_{1}>x_{2}>x_{3}>\cdots$. Introduce the inverse lexicographical order on the set $\mathcal{X}^{(n)}$ of all monomials in $\mathcal{X}$ of fixed degree $n$ : $\prod_{x} x^{i_{x}}<\prod_{x} x^{j_{x}}$, where $\sum_{x} i_{x}=\sum_{x} j_{x}=n$, if there is $x_{0} \in \mathcal{X}$ such that $i_{x}=j_{x}$ for all $x<x_{0}$ and $i_{x_{0}}>j_{x_{0}}$. It is easy to see that this is a complete order also.

Lemma. Let $W$ be a vector space spanned by a completely ordered set of its vectors $w \in \mathcal{W}$. Then the set of all $w \in \mathcal{W}$ which cannot be expressed as a (finite) linear combination of the smaller ones forms a basis of $W$.

Applying this statement to the space $W=A_{n}$ generated by the images of monomials of degree $n$, we obtain a monomial basis $S_{n} \subset \mathcal{X}^{(n)}$ in $A_{n}$. It is clear that any monomial of degree $k$ which divides a monomial from $S_{n}$ belongs to $S_{k}$. This basis of $A$ is called a commutative PBW-basis if the set $S_{n}$ coincides with the set of all monomials of degree $n$ whose divisors of degree 2 belong to $S_{2}$. It is easy to see that any algebra admitting a commutative $\mathrm{PBW}$-basis is quadratic.

Remark. In the case of a (skew-)commutative algebra, the notion of a commutative PBW-basis is more general then the well-known Priddy's definition [15] of a (non-commutative) PBW-basis. The following results are completely analogous to the non-commutative case.

Proposition. Assume that the $P B W$ condition above is satisfied for the monomial basis $S_{3}$ in the third degree component $A_{3}$ of a quadratic algebra $A$. Then the same is true for any degree $n$. Furthermore, a quadratic algebra admitting a commutative PBW-basis is Koszul.

Proof. The proof can be found in [14]. The first statement is well-known [5, 6]. The second one was proved in [9] using the result of R. Fröberg [7]. Some of these papers deal with the finite dimensional case, but this is not essential here.

A.2. Finite fields. Since $K_{2}^{\mathrm{M}}(F)=0$ for any finite field $F$ [12], this ring is evidently Koszul. 
A.3. The field of rational numbers. Let us show that the algebra $K^{\mathrm{M}}(\mathbb{Q}) \otimes \mathbb{F}_{l}$ is Koszul for any prime number $l$. (This will not be applicable to our problem directly since $G_{\mathbb{Q}}$ is not a pro-l-group for any $l$.) By the well-known results of J. Milnor [13] and H. Bass and J. Tate [3], one has

$$
K_{n}^{\mathrm{M}}(\mathbb{Q}) \simeq \bigoplus_{\text {prime } p} K_{n-1}^{\mathrm{M}}\left(\mathbb{F}_{p}\right) \bigoplus K_{n}^{\mathrm{M}}(\mathbb{R}) \otimes \mathbb{F}_{2},
$$

where $\partial_{p}: K_{n}^{\mathrm{M}}(\mathbb{Q}) \longrightarrow K_{n-1}^{\mathrm{M}}\left(\mathbb{F}_{p}\right)$ is the boundary homomorphism. Explicitly, we have

$$
\begin{aligned}
& K_{2}^{\mathrm{M}}(\mathbb{Q}) \otimes \mathbb{F}_{l} \simeq \bigoplus_{\text {prime } p>2} \mathbb{F}_{p}^{*} /\left(\mathbb{F}_{p}^{*}\right)^{l} \oplus \begin{cases}\mathbb{Z} / 2 \mathbb{Z}, & \text { for } l=2 \\
0, & \text { for } l>2,\end{cases} \\
& K_{n}^{\mathrm{M}}(\mathbb{Q}) \otimes \mathbb{F}_{l} \simeq\left\{\begin{array}{ll}
\mathbb{Z} / 2 \mathbb{Z}, & \text { for } l=2 \\
0, & \text { for } l>2,
\end{array} \text { for } n \geqslant 3,\right.
\end{aligned}
$$

where the multiplication is given by the formulas

$$
\begin{aligned}
& \{a, b\}_{p}=(-1)^{\nu_{p}(a) \nu_{p}(b)} \frac{a^{\nu_{p}(b)}}{b^{\nu_{p}(a)}} \bmod p \\
& \left\{a_{1}, \ldots, a_{n}\right\}_{\infty}=\left\{\begin{array}{lll}
1 & \bmod 2, & \text { if } a_{1}, \ldots, a_{n}<0 \\
0 & \bmod 2, & \text { otherwise }
\end{array}\right.
\end{aligned}
$$

Let us construct PBW-bases for these algebras in an explicit way. Consider the two cases separately.

(a) $l=2$. The vector space $K_{1}^{\mathrm{M}}(\mathbb{Q}) \otimes \mathbb{F}_{2}$ admits a basis consisting of the symbols $\{p\}$, for all prime numbers $p$, and $\{-1\}$. Let $R$ be the set of all odd primes $r$ for which 2 is a square modulo $r$ and $Q$ be the set of all other odd primes $q$. Fix arbitrary complete orders on the sets $Q$ and $R$ and extend them to an order on the whole basis by the rule $\{2\}<\{q\}<\{r\}<\{-1\}$ for any $q \in Q$ and $r \in R$. For each $r \in R$, let $q(r)$ denote the minimal prime $q \in Q$ with respect to the order which is not a square modulo $r$. Since $\{2,2\}=\{2, r\}=0$ for any $r \in R$, it is easy to verify that the monomials $\{2, q\},\{q(r), r\}$, and $\{-1, \ldots,-1\}$ form the basis of $K^{\mathrm{M}}(\mathbb{Q}) \otimes \mathbb{F}_{2}$ corresponding to the order chosen. The PBW condition is clearly satisfied.

(b) $l>2$. The vector space $K_{1}^{\mathrm{M}}(\mathbb{Q}) \otimes \mathbb{F}_{l}$ admits a basis consisting of the symbols $\{p\}$ for all prime numbers $p$. Let $R$ be the set of all primes $r$ for which $r-1$ is divisible by $l$ and $Q$ be the set of all other primes $q$. Choose a complete order on our basis such that $\{q\}<\{r\}$ for any $q \in Q$ and $r \in R$. For each $r \in R$, let $q(r)$ denote the minimal $q \in Q$ which is not a $l$-th power modulo $r$. Since $\left\{q, q^{\prime}\right\}=0$ and $\{q, r\}_{q}=0$ for any $q$, 
$q^{\prime} \in Q$ and $r \in R$, it is easy to see that the monomials $\{q(r), r\}$ form a commutative PBW-basis of $K^{\mathrm{M}}(\mathbb{Q}) \otimes \mathbb{F}_{l}$.

\section{Acknowledgement}

We are grateful to V. Voevodsky for stating the problem and numerous stimulating discussions and to J. Bernstein who pointed out to us the necessity of using coalgebras in the Koszul duality. The first author is pleased to thank Harvard University for its hospitality which made it possible for this work to appear.

\section{References}

1. J. Backelin, A distributiveness property of augmented algebras and some related homological results, Ph. D. Thesis, Stockholm, 1981.

2. J. Backelin and R. Fröberg, Koszul algebras, Veronese subrings and rings with linear resolutions, Rev. Roumaine Math. Pures Appl. 30 (1985), 85-97.

3. H. Bass and J. Tate, The Milnor ring of a global field, Lecture Notes in Math. 342 (1973), 349-446.

4. A. A. Beilinson, V. A. Ginzburg and V. V. Schechtman, Koszul duality, Journ. Geom. Phys. 5, 317-350.

5. G. Bergman, The diamond lemma for rings theory, Advances in Math. 29 (1983), $178-218$.

6. B. Buchberger, Grobner bases: an algorithmic method in polynomial ideal theory, CAMP-Bull. 290 (1978).

7. R. Fröberg, Determination of a class of Poincare series, Math. Scand. 37 (1975), 29-39.

8. C. Löfwall, On the subalgebra generated by the one-dimensional elements in the Yoneda Ext-algebra, Lecture Notes in Math. 1183 (1986),291-338.

9. G. R. Kempf, Some wonderful rings in algebraic geometry, Journ. Algebra 134, p. 222-224, 1990.

10. A. S. Merkurjev and A. A. Suslin, K-cohomology of Severi-Brauer varieties and the norm residue homomorphism, Math. USSR Izvestiya 21 (1983), 307-340.

11. The norm residue homomorphism of degree three, Math. USSR Izvestiya 36 (1991), 349-367.

12. J. Milnor, Algebraic K-theory and quadratic forms, Invent. Math. 9 (1970), 318344.

13. Introduction to algebraic K-theory, Ann. Math. Studies, Princeton, 1971.

14. A. Polishchuk and L. Positselski, Quadratic algebras, in preparation.

15. S. Priddy, Koszul resolutions, Trans. AMS 152 (1970), 39-60.

16. M. Rost, Hilbert theorem 90 for $K_{3}$ for degree two extensions, preprint, 1986.

INDEPENDENT UNIVERSITY OF MOSCOW

E-mail address: posic@ium.ips.ras.ru,posic@math.harvard.edu

HARVARD UNIVERSITY

E-mail address: vishik@math.harvard.edu 